\title{
El sistema alemán de formación profesional (BERUFSAUSBILDUNG)
}

Óscar Javier Apáez Pineda *

\section{Resumen}

$\mathrm{C}^{1}$ propósito de este trabajo es explicar y analizar el sistema ¿alemán de formación profesional bajo una óptica jurídica, ya que, de conformidad con los datos estadísticos presentados por Alemania, en el pasado lustro se logró un 95\% de inserción laboral juvenil, en ese sentido nacen las interrogantes: ¿De qué manera se desarrolla y cómo funciona el sistema de formación profesional en Alemania?

Para dar respuesta a estas preguntas, se desarrolla una investigación documental, guiada por el método deductivo que parte de la descripción del sistema educativo alemán, continua con el análisis y descripción del sistema de formación profesional y la protección que se establece en el Código Social; por último, se ofrecen las reflexiones finales para su discusión.

\section{Palabras clave \\ Formación Profesional, Derecho Social, Educación.}

Fecha de recepción: octubre 2019
Fecha de aceptación:

noviembre 2019

\footnotetext{
* Doctor en Derechoy Globalización por la Universidad Autónoma de Morelos, Investigador Universidad La Salle, Ciudad de México. Miembro del Sistema Nacional de Investigadores, Candidato, CONACYT. oscar.apaez@lasalle.mx.
} 


\title{
German Professional Vocational Training System (BERUFSAUSBILDUNG)
}

\author{
Keywords \\ Vocational training, Social Law, Education. \\ Final submission: \\ October 2019 \\ Acceptance: \\ November 2019
}

\section{Abstract:}

The purpose of this paper is to explain and analyse the German system of vocational training, under a legal perspective, since in accordance with the statistical data presented by Germany in the past 2015 achieved $95 \%$ of youth employment insertion which let us raise the following questions: How is the vocational training system developed? And How does the vocational training system works in Germany? To respond this question, a documentary research is developed, guided by the deductive method that starts from the description of the German education system, continues with the analysis and description of the vocational training system with the support established in the Social Code, and finally, the last reflections are offered for discussion.

\section{Introducción}

Alemania tiene reconocido datos muy altos en materia laboral, por ejemplo, en el lustro pasado reportó que en relación con el desempleo, se tenía una tasa del $4.5 \%$ del total de la población, de este porcentaje aproximadamente el 7.6\% lo constituyen jóvenes lo que representa un $4.3 \%$ de los jóvenes, por lo que un $95.7 \%$ de estos tienen trabajo formal. ${ }^{1}$ De estos, tan solo el $1.8 \%$ permanecen desempleados por más de doce meses. ${ }^{2}$

Mientras que en materia de educación se reportaron tasas de deserción muy bajas en sus niveles básicos, pues al menos el $88.9 \%$ de los alemanes tiene estudios de primaria y secundaria.

1 EUROSTAT, Youth unemployment by sex, age and educational attainment level, disponible en: http://ec.europa.eu/eurostat/web/youth/data/database, fecha de consulta 12 de octubre de 2015.

2 Idem. 
En lo que hace a la deserción escolar por condiciones de maternidad o de inserción laboral el porcentaje fue del 9.5\%. Además, de que un $2.8 \%$ de los jóvenes que no se encuentran en educación formal, se encuentra realizando un entrenamiento o cursando educación no formal.

Datos que nos hacen reflexionar sobre la efectividad del sistema y sobre los pilares en los cuales se sostiene, principalmente el pilar educativo.

$\mathrm{Al}$ respecto, se precisa que poder analizar el sistema de formación profesional alemán, es necesario explicar el sistema educativo desde sus niveles iniciales, ya que eso nos permitirá dimensionar la importancia del seguimiento, normas y políticas educativas que logran niveles de inserción laboral precitados, por ello es que a continuación se explicará brevemente el sistema educativo para posteriormente, indicar las características y particularidades de la protección que se brinda en el proceso de formación profesional.

\section{Breve esbozo del sistema educativo alemán}

En la mayoría de los Estados Federados de Alemania, ${ }^{3}$ la educación inicia con la preprimaria, (Kindergarten) misma que dura 3 años y engloba a los menores que tienen entre 3 y 5 años, posterior a ello se tiene la Escuela Básica (Grundschule), que sería el equivalente a la educación primaria en México, solo que en Alemania abarca del primer al cuarto grado; a esta asisten los menores entre los 6 y 10 años:

Sistema alemán de educación básica

\begin{tabular}{|c|c|c|c|}
\hline Grado & Escuela & Edad & En México \\
\hline 4 & Grundschule & 9 & Primaria \\
\hline 3 & & 8 & \\
\hline 2 & & 7 & \\
\hline 1 & & 6 & \\
\hline Grado & Escuela & Edad & En México \\
\hline & Kindergarten & 5 & Pre-escolar \\
\hline
\end{tabular}

3 Con excepción de algunas ciudades como Berlín y Brandemburgo. 


\begin{tabular}{|l|l|l|l|}
\hline & & 4 & \\
\hline & & 3 & \\
\hline
\end{tabular}

Elaboración propia con datos de la Fuente: Kulturminister Konferenz, Das Bildungswesen in der Bundesrepublik Deutschland. ${ }^{4}$

Al terminar la primaria, continúa lo que en México denominamos educación secundaria, misma que dependiendo de la escuela en la cual se ingrese será su duración.

De acuerdo a las opciones de desarrollo del menor, en ese sentido tenemos cuatro opciones:

a) La Escuela Secundaria Básica, (Hauptschule) que comprende los grados quinto a décimo, con una duración de 6 años, esta se enfoca en preparar a los estudiantes fundamentalmente en oficios manuales (Handwerk), para ello proporciona conocimientos similares a las impartidos en la escuela secundaria (Realschule) y el bachillerato (Gymnasium) además de prácticas de los oficios.

Al concluir esta etapa se obtiene el Certificado de Educación Secundaria (Mittele reife) y se puede pasar a otro tipo de escuela de la siguiente etapa, aunque dada la formación, lo normal es ingresar directo a la Escuela de Formación Profesional (Berufsschule) y esto provoca que no se pueda acceder a la educación universitaria.

b) La Escuela Secundaria Técnica (Realschule) comprende los grados de quinto a décimo y dura 6 años, su objetivo es una formación para oficios de mayor cualificación técnica.

Una vez obtenido el certificado de secundaria existe la posibilidad de proseguir los estudios en las distintas opciones que brinda el sistema educativo en la etapa superior, por lo general se continúan los estudios en la Escuela de Técnica de Formación Profesional (Berufsfachschule), pues esta permite el acceso a Escuelas Técnicas de Ciencias Aplicadas.

4 Kulturminister Konferenz, Das Bildungswesen in der Bundesrepublik Deutschland 2014/2015, Disponible en:https://www.kmk.org/dokumentation-und-statistik/informationen-zum-deutschen-bildungssystem/dossier-deutsch.html, fecha de consulta 12 de octubre de 2017. 
c) La Escuela Secundaria Superior (Gymnasium), de desde el quinto grado al doceavo, tiene una duración de 8 años, en esta el alumno obtiene el grado de Bachillerato (Abitur) lo que le da posibilidad de elegir en que escuela de estudios superiores desea asistir, por lo general ingresan a la universidad sin necesidad de examen de ingreso.

d) La Escuela de Educación Secundaria Mixta (Gesamtschule), comprende los grados de quinto a décimo e imparte los conocimientos que normalmente se adquieren en la escuela técnica y la superior. Al finalizar los estudios se puede escoger cualquier otra escuela del siguiente nivel aunque por lo general los estudiantes continúan en la Escuela Técnica de Especialistas, (Fachoberschule).

Una vez que los jóvenes terminan este nivel educativo se tiene que escoger donde continuar sus estudios, esto equivale a la transición de educación secundaria a la media superior en México.

En este sentido tenemos las siguientes opciones:

a) La Escuela de Formación Profesional (Berufsschule), que comprende los grados décimo a décimo tercero, con duración de tres años, se obtiene el grado de Oficial (Geselle).

Al finalizar debe hacerse un grado de cualificación profesional que permite la posibilidad de ingresar a la Escuela de Maestros (Meisterschule).

b) La Escuela de Técnica de Formación Profesional (Beruffachschule), que comprende del grado décimo al décimo tercero, con duración de tres años, se obtiene el grado de Técnico (Thekniker).

Al finalizar debe hacerse un grado de cualificación profesional que permite la posibilidad de ingresar a la Escuela Profesional ( $\mathrm{Fa}$ chschule) o Escuelas Técnicas de Ciencias Aplicadas.

\footnotetext{
5 La traducción correcta de la palabra sería bachillerato, sin embargo, al tratarse de un segundo nivel educativo diversos autores manejan el término de escuela secundaria superior, en el siguiente nivel propiamente es el bachillerato, debido al tipo de formación que se imparte centrado en conocimientos propios de este como lo son literatura, filosofía etcétera. Véase: M. Gross, Dominique, Youth unemployment and youth labour market policies in Germany and Canada, International Labour Office, Geneva, 1998.
} 
c) El bachillerato (Gymnasium), comprende el grado décimo primero al décimo tercero, al finalizar se obtiene el grado de bachiller (Abitur) y permite ingresar a la Universidad (Universität), la escuela superior (Hochschule) o la academia (Akademie).

d) Escuela Técnica de Especialistas, (Fachoberschule), comprende los grados décimo primero y décimo segundo, al finalizar se obtiene el grado de Bachiller Especializado (Abitur) ya que los estudios que se imparten son en un área específica de las ciencias sociales.

Una vez concluida esta etapa se cuenta con tres opciones más:

a) La Escuela de Maestros (Meisterschule), ofrece el grado de maestro (Maiester) para aquellos estudiantes con grado de oficial.

b) La Escuela Profesional (Fachschule) o Escuelas Técnicas de Ciencias Aplicadas, ofrece el grado de licenciatura (Bachelor) a los alumnos con el grado de técnicos (Thekniker).

c) La Universidad (Universität), la Escuela Superior (Hochschule) o la Academia (Akademie), ofrecen el grado de licenciatura (Bachelor), a los estudiantes con grado de bachiller (Abitur) o a los técnicos (Thekniker) que aprueban el examen de ingreso.

Para una mejor comprensión de lo señalado, se presenta un cuadro sinóptico que nos permite visualizar la organización de la educación en Alemania, además de permitirnos observar las diferencias con el sistema mexicano:

Sistema alemán de educación media y superior

\begin{tabular}{|c|c|c|c|c|c|c|}
\hline 葡 & & & & & Edad & $\begin{array}{c}\text { En } \\
\text { México }\end{array}$ \\
\hline & $\begin{array}{c}\text { Meisterschule } \\
\text { Escuela de } \\
\text { Maestros }\end{array}$ & $\begin{array}{c}\text { Fachschule } \\
\text { Escuela } \\
\text { Profesional }\end{array}$ & $\begin{array}{c}\text { Universität, } \\
\text { Hochschule, } \\
\text { Akademie } \\
\text { Universidad, } \\
\text { Escuela } \\
\text { Superior, } \\
\text { Academia }\end{array}$ & 19 & $\begin{array}{c}\text { Educación } \\
\text { Superior }\end{array}$ & \\
\hline
\end{tabular}




\begin{tabular}{|c|c|c|c|c|c|c|}
\hline & & & $\begin{array}{l}\text { Gymnasium } \\
\text { Bachillerato }\end{array}$ & & & \\
\hline 13 & $\begin{array}{c}\text { Cualificación } \\
\text { Profesional }\end{array}$ & $\begin{array}{c}\text { Cualificación } \\
\text { profesional }\end{array}$ & & & 18 & $\begin{array}{c}\text { Educación } \\
\text { media } \\
\text { superior }\end{array}$ \\
\hline 12 & \begin{tabular}{|c|} 
Berufsschule \\
Escuela de \\
Formación \\
Profesional
\end{tabular} & \begin{tabular}{|c|} 
Berufsfachschule \\
Escuela de \\
Técnica de \\
Formación \\
Profesional \\
\end{tabular} & & $\begin{array}{c}\text { Fachoberschule } \\
\text { Escuela } \\
\text { Técnica de } \\
\text { Especialistas }\end{array}$ & 17 & \\
\hline 11 & & & & & 16 & \\
\hline 10 & & & & & 15 & \\
\hline 10 & $\begin{array}{c}\text { Hauptschule } \\
\text { Escuela } \\
\text { Secundaria } \\
\text { Básica }\end{array}$ & $\begin{array}{l}\text { Realschule } \\
\text { Escuela } \\
\text { Secundaria } \\
\text { Técnica }\end{array}$ & $\begin{array}{l}\text { Gymnasium } \\
\text { Escuela } \\
\text { Secundaria } \\
\text { Superior }\end{array}$ & $\begin{array}{c}\text { Gesamtschule } \\
\text { Escuela de } \\
\text { Educación } \\
\text { Secundaria } \\
\text { Mixta }\end{array}$ & 15 & $\begin{array}{l}\text { Educación } \\
\text { Secundaria }\end{array}$ \\
\hline 9 & & & & & 14 & \\
\hline 8 & & & & & 13 & \\
\hline 7 & & & & & 12 & \\
\hline 6 & & & & & 11 & \\
\hline 5 & & & & & 10 & \\
\hline
\end{tabular}

Fuente: Kulturminister Konferenz, Das Bildungswesen in der Bundesrepublik Deutschland. ${ }^{6}$

La educación en Alemania es obligatoria por lo menos diez grados y a partir de ahí los jóvenes de 15 años podrán ingresar al mundo laboral o continuar con su preparación profesional; en el caso existe la formación dual profesional que puede iniciarse a esta edad en la Escuela de Formación Profesional (Berufschule) y en la Escuela de Técnica de Formación Profesional (Berufsfachschule). Además, a partir de los 18 años los jóvenes tendrán que culminar sus estudios o continuar sus oficios o sus carreras técnicas, para ello el sistema alemán cuenta con diferentes medidas de promoción, elección, orientación y fomento del trabajo, mismas que pueden aplicar a partir de los 10 años y que en el siguiente título se desarrollan.

6 Kulturminister Konferenz, op. cit. 
En lo que concierne a orientación o elección de profesión, a los 15 años de edad se prevén medidas de fomento y apoyo para obtener trabajo, pues la legislación social señala que a partir de esa edad se puede comenzar la vida laboral, a continuación, abordaremos el contenido del Código Social (Sozialgesetzbuch, SGB) respecto a las medias de apoyo para la formación profesional.

\section{Código Social (Sozialgesetzbuch, SGB)}

Este Código contiene en sí diversos libros que regulan lo que en el sistema jurídico alemán se entiende por Derecho Social, en ese sentido encontramos que regula aspectos de seguridad social, laboral e incluye a la formación profesional misma que analizamos en relación con lo contenido en dicho Código.

\section{Libro tercero relativo a la formación profesional ${ }^{7}$}

Es importante mencionar que por cuestiones históricas y tradicionales, la formación profesional constituye un pilar esencial de la economía del país pues los empleos para personas con mano de obra no cualificada o capacitada son muy reducidos, solo ante una irresponsabilidad total, un joven caería en desamparo, ya que la educación obligatoria es hasta el décimo grado; además de que se cuentan con las siguientes medidas:

\section{Acompañamiento para la iniciación profesional ${ }^{8}$}

Esta medida de protección consiste en un apoyo directo entre la escuela y formación profesional del joven en escuelas de nivel secundario con calificaciones no adecuadas o inexperiencia técnica para acceder al siguiente nivel de estudios ${ }^{9}$ o aquellos jóvenes migrantes.

Con el fin de lograr una mejor y mayor transición se cuenta con la Ley sobre la Mejora de Posibilidad de Inserción al Mercado

\footnotetext{
7 SGB III Buch Arbeitsförderung.

8 Berufseinstiegsbegleitung.

9 Por ejemplo: Un joven de la Escuela Secundaria Técnica que desea migrar a las Escuelas de Formación Profesional o del Bachillerato a una de estas escuelas.
} 
Laboral, (Gesetz zur Verbesserung der Eingliederungschancen am Arbeitsmarkt), que introduce el denominado Acompañamiento para la Iniciación Profesional, (Berufseinstiegsbegleitung). Este consiste en que jóvenes de niveles más altos de formación profesional acompañan a los jóvenes con limitantes en su iniciación profesional de forma individual y permanente hasta completar el ciclo de enseñanza general básica o de una escuela de educación especial hasta unos seis meses desde la clase pre-terminal del décimo grado.

Estos acompañantes ayudan a la reducción de déficits de lenguaje y educación, la promoción de la teoría de la técnica y un apoyo socio-educativo para la gente joven rezagada.

En el caso de que la transición no se logre de forma inmediata, el acompañamiento se puede extender también a la fase de transición, hasta el décimo grado hasta un máximo de 24 meses después de terminar los estudios escolares, es decir, el joven permanecerá acompañado durante este tiempo para poder lograr su transición a la educación media superior. Este apoyo es financiado por los gobiernos estatales y el gobierno federal, pero la ley contempla la posibilidad de obtener financiamiento de terceros. ${ }^{10}$

\section{Medidas preparatorias de formación profesional}

A discreción de las agencias locales de empleo el SGB permite que se fomente la formación preparatoria de jóvenes que no han iniciado su formación profesional, estas medidas pueden incluir además de la respectiva orientación e identificación de profesiones, una formación preparatoria para el aprendizaje.

En el marco de estas medidas se establece el derecho al fomento de preparación necesaria para que quienes no cuenten con la preparación básica obligatoria culminen sus estudios y obtengan su diploma de educación secundaria.

${ }^{10}$ Debido a la imposibilidad de cofinanciación a corto plazo para muchos Estados Federados, el Gobierno Federal ha asegurado la cofinanciación con fondos del Fondo Social Europeo. 


\section{El sistema de formación dual profesional (Duale Ausbildung)}

El origen de este sistema se remonta a los gremios de artesanos alemanes del siglo XII, quienes estructuraron un modelo de formación aprendiz-oficial-maestro y crearon la especialización en sus oficios.

Además, para la segunda mitad del siglo XIX, las primeras empresas industriales implantaron talleres de aprendices con su correspondiente formación laboral.

Con base en ello, se emitió el Reglamento Industrial de 1869, que por primera vez introdujo la enseñanza laboral obligatoria para los trabajadores menores de 18 años. Con la promulgación de la Ley de Protección del Artesanado de 1897 (Handwerkerschutzgesetz) se incluyó en el principio dual de formación laboral, y nació la formación práctica en la empresa junto a las clases teóricas en la escuela.

A principios del XX, las empresas introdujeron las profesiones comerciales y nace en Prusia (1923), el concepto de Escuela de Formación (Berufsschule).

Con la Ley de Formación Profesional (Berufsbildungsgesetz $B B i G)$ de 1969 , las diferencias regionales se unificaron en una regulación común. Así, la ley pretendía recoger las necesidades del Estado, los empresarios y los trabajadores. La ley también prevé la estrecha colaboración de los interlocutores sociales en el desarrollo de la formación profesional, algo que se mantiene hasta hoy día.

El sistema de formación dual no forma parte del SGB, sino que tiene su fundamento legal en la BBiG, reformada en el año 2005 y actualmente vigente, este constituye desde 1960 la principal medida de fomento y protección en la transición laboral de los jóvenes alemanes al mundo laboral.

De conformidad con la sección 1 , párrafo 3 de la $\mathrm{BBiG}$, la formación profesional dual debe otorgarse a través de un entrenamiento sistemático, que programe e imparta la formación profesional, conocimientos, calificaciones, competencia profesional y lo necesario para participar en como profesional calificado, ello además de permitir que los alumnos adquieran la experiencia necesaria para el trabajo. 
De ahí que este sistema reciba su nombre pues se trata de un programa a través del cual se busca que los jóvenes al asistir a la educación media superior obtengan los conocimientos teóricos necesarios para el ejercicio de una profesión u oficio y a su vez tengan los conocimientos prácticos en un centro de trabajo e incluso una remuneración por sus servicios. Es decir una formación dual escuela-trabajo, conocimiento teórico y práctico.

Son elegibles para este programa los jóvenes que cursan sus estudios en la Escuela de Formación Profesional (Berufsschule) y la Escuela de Técnica de Formación Profesional (Berufsfachschule). Situación que provoca que un gran número de jóvenes ingresen al mercado laboral y decidan al obtener su título de oficial o técnico, interrumpir su formación y seguir en el mundo laboral.

Respecto a la cantidad de jóvenes entre 10 y 15 años, que eligen una escuela de formación de oficios o técnica, asciende a 2,508,678 hasta el año 2015, esto representa un 50\% de los jóvenes entre 15 y 18 años. ${ }^{11}$

Para finales del año 2015 se reportó que aproximadamente 448,006 jóvenes se encontraban sujetos al sistema de formación dual, mismos representa cerca del $50 \%$ de jóvenes que en escuelas profesionales deciden adelantar su vida profesional y su inserción laboral a partir de los 15 años.

Con base en lo anterior las estadísticas nos refieren que el 50\% de los actuales trabajadores alemanes provienen de este sistema de formación profesional. ${ }^{12}$

Por lo anterior es que es necesario precisar la organización, funcionamiento y relación de dicho sistema con el sistema educativo mismo que a continuación abordaremos.

\footnotetext{
${ }^{11}$ Statistisches Bundesamt, Schools of general education and vocational schools, https://www.destatis.de/EN/FactsFigures/SocietyState/EducationResearchCulture/ Schools/Tables/PupilsSchoolType.html, Fecha de consulta 12 de octubre de 2015.

12 Bildungsstand der Bevölkerung 2015 (destatis) - Estado Formativo de la Población Alemana 2015, disponible en línea en: https://www.destatis.de/DE/ Publikationen/Thematisch/BildungForschungKultur/Bildungsstand/BildungsstandBevoelkerung.html, fecha de consulta: 15 de diciembre de 2016.
} 


\section{Organización}

Este programa cuenta con la participación de entidades gubernamentales y los empresarios del país. Por parte del Gobierno alemán el Ministro Federal de Educación e Investigación (Bundesministerium für Bildung und Forschung) participa como el responsable general de la formación profesional y es quien fija y desarrolla los criterios del sistema dual de desarrollo profesional.

Además ofrece apoyo a las empresas a efectos de desarrollar los centros de promoción de formación profesional, promueve programas de innovación profesional y flexibilidad, programas para la inserción de persona con capacidades diferentes y promociona la investigación y el desarrollo de los proyectos relativos a la formación dual. Para materializar este programa es necesaria la participación de las empresas y escuelas no solo en planos teóricos sino en armonía práctica tal y como a continuación se explica.

\section{Funcionamiento}

El sistema de formación dual se desarrolla la mayor parte en la empresa y las escuelas tan solo actúan como soporte teórico técnico de las habilidades de los alumnos, en cada uno de estos lugares se cuenta con propios reglamentos (Ausbildungsordnung), lo que hace que el joven mantenga un contacto directo con la forma de vida de un profesional.

En este sistema el joven pasa 3 o 4 días a la semana en la empresa, las actividades que desarrollan en las empresas se basan en reglamentos de entrenamiento que a su vez tienen su fundamentación en un contrato que la empresa y el joven pactan. ${ }^{13}$ Este contrato debe contener por lo menos las siguientes aspectos:

- Reconocimiento del estado de la ocupación, es decir, definición precisión qué es lo que se va a realizar,

- Designación de la ocupación, categoría, puesto y denominación,

- Duración de la formación,

${ }^{13}$ Sección 4, párrafo 2, Ley de formación profesional, BBiG. 
- Perfil de la ocupación del entrenamiento,

- Plan de formación general, es decir esquema del itinerario y el horario,

- Los requisitos de evaluación del desempeño del alumno.

Por lo general estos contratos señalan que el período de formación es el estipulado en la Ley 2 a 3 años dependiendo del desempeño y necesidades. $\mathrm{Al}$ respecto la duración de formación puede ser acortada o extendida bajo ciertas condiciones que deberán justificarse por la empresa.

Además las empresas deben presentar los contenidos de la formación y un plan de capacitación mismo que se considera parte del contrato y forma parte de este como anexo.

Para la rescisión del contrato: los alumnos cuentan con protección contra el despido si es que sus periodos de pruebas o formación concluyen. ${ }^{14}$ Por lo que son considerados como trabajadores de la empresa.

En relación al funcionamiento del sistema, las empresas ofertan plazas de educación formativa junto con el contrato respectivo. Además establecen un tutor que será encargado de la formación del joven en la empresa, durante la estancia en la empresa recibe la denominación de aprendiz.

Los días de la semana que corresponden a la formación teórica pueden ser 1 o 2 de la semana, esto se debe a que esta formación se encuentra contemplada en planes de estudio (Rahmenlehrplan), previamente aprobados por el ministerio, mismos que deben de proporcionar conocimientos especializados y los conocimientos básicos del marco de educación obligatoria.

Este plan de estudios contempla dos tercios de contenido de programas con enfoque basado en la actividad del campo de aprendizaje que desarrolla el joven y un tercio reservado a la formación general.

La cantidad de planes de estudio es muy variada pues no solo depende de los oficios o profesiones que se desarrollan, sino que depende también del tipo de empresa y sobre todo de los planes aprobados por

${ }^{14}$ Véase las medidas referidas en el título que precede. 
cada una de las entidades federativas, pues es su competencia el desarrollo de estos planes educativos, por lo que su vínculo con el sistema educativo es evidente y a continuación se desarrolla. ${ }^{15}$

\section{Relación con el sistema educativo}

El sistema de formación dual impacta el sistema educativo por lo que existen alumnos que asisten a escuelas vocacionales de tiempo completo, es decir, dentro de las escuelas de formación técnica profesional y la de oficios, que se encuentran sombreadas en la siguiente tabla:

\begin{tabular}{|c|c|c|c|c|c|c|c|}
\hline 율 & & & & & & 苞 & $\begin{array}{c}\text { En } \\
\text { México }\end{array}$ \\
\hline & $\begin{array}{l}\text { Meisterschue } \\
\text { Escuela } \\
\text { de } \\
\text { Maestros }\end{array}$ & \multirow{5}{*}{$\begin{array}{l}\text { Escuelas } \\
\text { de oficio y } \\
\text { profesionales } \\
\text { de tiempo } \\
\text { completo }\end{array}$} & \multirow[t]{2}{*}{$\begin{array}{l}\text { Fachschule } \\
\text { Escuela } \\
\text { Profesional }\end{array}$} & \multirow{2}{*}{\multicolumn{2}{|c|}{$\begin{array}{c}\text { Universität, Hochschule, } \\
\text { Akademie } \\
\text { Universidad, Escuela } \\
\text { Superior, Academia }\end{array}$}} & & $\begin{array}{l}\text { Educación } \\
\text { Superior }\end{array}$ \\
\hline 13 & & & & & & 18 & \multirow{4}{*}{$\begin{array}{c}\text { Educación } \\
\text { media } \\
\text { superior }\end{array}$} \\
\hline 12 & \multirow[t]{3}{*}{$\begin{array}{l}\text { Berufsschule } \\
\text { Escuela de } \\
\text { Formación } \\
\text { Profesional }\end{array}$} & & \multirow{3}{*}{$\begin{array}{l}\text { Berufs- } \\
\text { fachschule } \\
\text { Escuela de } \\
\text { Técnica de } \\
\text { Formación } \\
\text { Profesional }\end{array}$} & \multirow[t]{3}{*}{$\begin{array}{l}\text { Gymnasium } \\
\text { Bachillerato }\end{array}$} & \multirow{3}{*}{$\begin{array}{l}\text { Fachober- } \\
\text { schule } \\
\text { Escuela } \\
\text { Técnica de } \\
\text { Especialistas }\end{array}$} & 17 & \\
\hline 11 & & & & & & 16 & \\
\hline 10 & & & & & & 15 & \\
\hline \begin{tabular}{|l|}
10 \\
\end{tabular} & \multirow{6}{*}{\multicolumn{2}{|c|}{$\begin{array}{c}\text { Hauptschule } \\
\text { Escuela Secundaria } \\
\text { Básica }\end{array}$}} & \multirow{6}{*}{$\begin{array}{l}\text { Realschule } \\
\text { Escuela } \\
\text { Secundaria } \\
\text { Técnica }\end{array}$} & \multirow{6}{*}{$\begin{array}{l}\text { Gymnasium } \\
\text { Escuela } \\
\text { Secundaria } \\
\text { Superior }\end{array}$} & \multirow{6}{*}{$\begin{array}{l}\text { Gesamtschule } \\
\text { Escuela de } \\
\text { Educación } \\
\text { Secundaria } \\
\text { Mixta }\end{array}$} & 15 & \multirow{6}{*}{$\begin{array}{l}\text { Educación } \\
\text { Secundaria }\end{array}$} \\
\hline 9 & & & & & & 14 & \\
\hline 8 & & & & & & 13 & \\
\hline \begin{tabular}{|l}
7 \\
\end{tabular} & & & & & & 12 & \\
\hline 6 & & & & & & 11 & \\
\hline 5 & & & & & & 10 & \\
\hline
\end{tabular}

Kulturminister Konferenz, Das Bildungswesen in der Bundesrepublik Deutschland. ${ }^{16}$

Como se observa en la tabla, las escuelas de nivel medio superior que permiten el sistema de formación profesional, son solo las que forman a los oficiales y técnicos, siendo importante resaltar que una característica del sistema de formación profesional es la remuneración por los servicios prestados en la formación profesional, en este sentido los jóvenes son considerados trabajadores de

${ }^{15}$ Estos planes se aprueban por la Conferencia de Ministros Estatales de Educación y Temas Culturales (Kultusministerkonferenz).

${ }^{16}$ Kulturminister Konferenz, op. cit. 
conformidad con SGB que señala que el trabajo solo puede realizarse a partir de los 15 años.

Para los jóvenes en los niveles inferiores o aquellos que no concluyeron y se ven imposibilitados para integrarse en el sistema dual, aplican las medidas mencionadas en el título que precede sin que les sea aplicable el sistema dual.

Además de lo aludido en Alemania existe la figura de ocupación con reconocimiento de entrenamiento (Anerkannter Ausbildungsberuf) que se trata de ocupaciones y oficios que por Ley requiere entrenamiento formal dentro del sistema dual para ejercerse.

En relación con ello hay 348 ocupaciones u oficios de este tipo de los cuales resaltan las ocupaciones clásicas: como operador industrial, chofer, secretaria ejecutiva, carnicero, panadero, artesano y agricultor, resaltando aquellas que por el desarrollo de la ciencia y la sociedad son novedosas como: especialista en investigación social y mercado (Fachangestellter für Marktund Sozialforschung), asistente de ingeniero civil (Geomatiker Geomatikerin), operador de máquinas y plantas (Maschinen und Anlagenführerin), especialista en seguridad y protección (Fachkraft für Schutz und Sicherheit), técnico en reciclaje y gestión de residuos (Fachkraft für Kreislaufund Abfallwirtschaft), administrativo de servicios de salud (KaufmannKauffrau im Gesundheitswesen) y microtecnólogo (Mikrotechnologe Mikrotechnologin). Oficios que son especializados y que cuentan con un alto grado de demanda empresarial.

Es también importante mencionar que los alumnos que optan por este tipo de formación profesional obtienen un certificado que les permite realizar estudios superiores en las respectivas escuelas superiores de oficios o técnicas, además de la posibilidad de realizar un examen de ingreso a universidad e incluso permite solicitar trabajo en una empresa distinta.

Para evitar situaciones ilegales de ingreso y promoción de los jóvenes dentro de sistema, a continuación, se precisa la manera en la cual se supervisa y se toman las decisiones trascendentales del mismo. 


\section{Administración, supervisión y financiamiento}

Uno de los aspectos fundamentales que ha permitido que el sistema continúe desde los años sesenta tiene que ver con la división de responsabilidades del sistema y la participación es su desarrollo, en este sentido, se encuentra que los avances más importantes en formación dual se discuten conjuntamente por el Gobierno Federal, los Estados Federados, los sindicatos, y la industria, mismos que a través del consenso comparten y desarrollan sus responsabilidades estos participantes del sistema constituyen el Comité de Federal de Educación y Formación Profesional ${ }^{17}$ quien se encarga de administrar todo el sistema de formación dual.

En ese sentido, corresponde al Gobierno Federal el reconocimiento y otorgamiento de diplomas, la determinación de ocupaciones con entrenamiento dual obligatorio, determinar los requisitos de formación y exámenes, elaborar el Reglamento de capacitación y promover las medidas de apoyo e investigación de la formación dual profesional.

Corresponde a los Estados Federados, diseñar los planes de estudio, financiar la infraestructura y pago de maestros requeridos y supervisar las actividades de los empresarios.

Por su parte, los empresarios y sindicatos elaboran propuestas para la creación de nuevas ocupaciones y la actualización de las existentes, nombran a expertos para participación en la redacción del Reglamento de Capacitación y llevan a cabo la negociación del importe del subsidio a alumnos.

Las cámaras de industria asesoran a las partes interesadas en la formación, supervisan la capacitación en la empresa, verifican la aptitud de las empresas e instructores, llevan el registro de contratos de formación y administran exámenes.

Cabe señalar que el financiamiento en su mayor parte recae en las empresas o compañías que solicitan a los alumnos, pues son quienes pagan remuneraciones y las respectivas cuotas de seguridad social del aprendiz, aunque el gobierno a nivel federal aporta cantidades que dependen de la ocupación que se requiere fomen-

${ }^{17}$ Sección 76, párrafo 2, titulo 3, Ley de Formación Profesional. 
tar, además de otorgar incentivos fiscales a los patrones de conformidad con el porcentaje de alumnos graduados o en formación y por su parte como se mencionó, los Estados Federados absorben el costo de la infraestructura para la impartición de clases teóricas y el pago a los profesores.

Por último, de conformidad con el reporte de educación de 2014 , la gente que accede a la universidad ha aumentado y para el caso por primera vez desde 2011 los niveles entre jóvenes que ingresaron al sistema dual estuvieron casi al parejo con los de los ingresos a educación superior, lo cual significa que los jóvenes están yendo más allá de la formación profesional para aumentar su educación y sus ingresos. ${ }^{18}$ Esto también ha significado que cada vez sean menos los jóvenes alemanes con intereses en el sistema Dual y el sistema haya tenido que echar mano de jóvenes migrantes.

\section{Reflexiones finales}

Como se pudo observar el sistema de formación profesional en Alemania se basa en un sistema educativo que va formando a los estudiantes en atención a sus habilidades y competencias, además de contar con el apoyo del Derecho Social; que intenta disminuir los riegos a los cuales se exponen los estudiantes durante su formación.

El impacto que los acontecimientos históricos han ejercido en la legislación educativa y laboral alemana puede observarse en todas y cada una de las medidas que aquí se analizan, aunque si bien es cierto, algunas no se materializan de forma significativa, el sistema formación profesional a través de la educación dual es uno de los pilares en la formación de los jóvenes alemanes y pieza fundamental para conservar los niveles de empleabilidad reportados hasta el año 2015.

Aunado a ello, a través de este sistema se asegura una transición casi en automático al mundo laboral, de hecho, elegir una formación profesional en este sistema convierte al joven en un auténtico trabajador.

18 Bundesministerium für Bildung und Forschung, Education Report 2014, disponible en: https://www.bmbf.de/publikationen/, fecha de consulta 12 de octubre de 2015. 
Es muy polémico el tema relativo al tipo de empleo o trabajo que ofrece el sistema dual, ya que actualmente, las categorías de oficios y profesiones técnicas que ofrece el sistema, no gozan del prestigio de una carrera universitaria.

Al respecto y en comparación con el sistema educativo en México y nuestro propio sistema de formación dual, resalta que la necesidad de que al igual que el sistema alemán nuestro sistema educativo contemple la posibilidad de tener estudiantes como auténticos trabajadores, situación que en el sistema alemán constituye una fortaleza.

Uno de los retos más grande para nuestro país en este sentido es el que tiene que ver con aspectos financieros, mismo que guarda relación con la inversión en la formación de sus jóvenes, pues México no cuenta con una tradición de inversión en capital humano y contrario a lo que Alemania nos muestra, las inversiones en educación y promoción de formación profesional en nuestro país no son primordiales.

Otra diferencia que podemos apuntar del sistema dual, son sus raíces en la Edad Media, pues se basa en la idea del antiguo funcionamiento de los talleres gremiales, y los datos estadísticos nos demuestran la madurez del sistema además de las grandes ventajas de inserción laboral y formación adecuada a los fines del Estado; aunque siempre queda la crítica respecto a que dicha formación puede atender a intereses económicos de las empresas involucradas y no de formación integral de la persona.

En el caso de México, la implementación de un sistema dual de inserción laboral no es factible tal y como lo tienen los alemanes, sobre todo porque reconocer la categoría de trabajador del estudiante, implica el gran reto de lograr el consenso de los principales actores sociales: empresas, sindicatos, gobierno estatal, federal, e instituciones educativas, situación que se vislumbra como un proceso largo, ríspido y con opiniones polarizadas.

Por último, debemos apuntar que además de la formación profesional que Alemania otorga a sus jóvenes y las medidas previstas para evitar la deserción escolar y promover la inserción, se cuenta con una legislación social complementaria de protección de riesgos específicos de los jóvenes, misma que ha provocado que los 
números de deserción y desempleo estén en niveles muy bajos, se cumple así el propósito de ser un sistema de formación profesional exitoso en los rubros señalados.

\section{Fuentes}

\section{Bibliohemerografía}

Blasco López Andreu y Jesús Hernández Aristu (comps.) Jóvenes más allá del empleo. Estructuras de apoyo a las transiciones de los jóvenes, Editorial Nau Libres, España, 2001.

Cacerés-rebbs, Diana y Scheider, Udo, "Vocational education and training in Mexico - how the country has tropicalised the dual model", Zeitschrift Des Bundesinstituts Für Berufsbildung, Verlag, núm. 5, Octubre 2015.

Cavalli, Alessandro y Galland, Olivier, Youth in Europe, Londres, Pinter, 1995.

M. Gross, Dominique, Youth unemployment and youth labour market policies in Germany and Canada, International Labour Office, Geneva, 1998.

Palos, Enrique y Herráiz, Marc, "El sistema de educación dual: nuevas avenidas en la cooperación bilateral entre Alemania y México", Revista Mexicana de Política Exterior, México, núm. 99, Sep-Dic 2013.

Sánchez Castañeda (editor) Youth Unemployment and Joblessness, Causes, Consequences, Responses, Ed. Cambridge Scholars, UK, 2012.

\section{Documentos electrónicos}

Bildungsstand der Bevölkerung 2015 (destatis) - Estado Formativo de la Población Alemana 2015, disponible en línea en: https://www.destatis.de/DE/Publikationen/Thematisch/ BildungForschungKultur/Bildungsstand/BildungsstandBevoelkerung.html, fecha de consulta: 15 de diciembre de 2019.

Bundesministerium Für Bildung und Forschung, Education Report 2014, disponible en: https://www.bmbf.de/publikationen/, fecha de consulta 12 de octubre de 2019. 
EUROSTAT, Youth unemployment by sex, age and educational attainment level, disponible en: http://ec.europa.eu/eurostat/web/ youth/data/database, fecha de consulta 12 de octubre de 2019.

Kulturminister Konferenz, Das Bildungswesen in der Bundesrepublik Deutschland 2014/2015, Disponible en:https:// www.kmk.org/dokumentation-und-statistik/informationen-zum-deutschen-bildungssystem/dossier-deutsch.html, fecha de consulta 12 de octubre de 2019.

Statistisches Bundesamt, Schools of general education and vocational schools, https://www.destatis.de/EN/FactsFigures/SocietyState/EducationResearchCulture/Schools/Tables/PupilsSchoolType.html, Fecha de consulta 12 de octubre de 2019.

\section{Legislación}

Código Social (Sozialgesetzbuch, SGB)

Ley de Formación Profesional (Berufsbildungsgesetz BBiG)

Ley de Protección del Artesanado de 1897 (Handwerkerschutzgesetz)

Ley del Impuesto sobre la Renta (Einkommensteuergesetz)

Ley Federal de Subsidio Familiar por Hijos (Bundeskindergeldgesetz)

Ley Fundamental para la República Federal Alemana (Grundgesetz für die Bundesrepublik Deutschland, GBD)

Ley sobre la Mejora de Posibilidad de Inserción al Mercado Laboral, (Gesetz zur Verbesserung der Eingliederungschancen am Arbeitsmarkt) 